\title{
Plasma Resistivity Determination in Runaway Discharges From Positive Voltage Spikes on TCABR Tokamak
}

\author{
Yu. K. Kuznetsov, I. C. Nascimento, R. M. O. Galvão and V. S. Tsypin \\ Instituto de Física, Universidade de São Paulo, \\ C.P. 66318, 05315-970 São Paulo, Brazil
}

Received on 26 June, 2001

\begin{abstract}
Plasma resistivity is estimated in runaway discharges on TCABR tokamak from the decay of positive voltage spikes using method developed in I. El Chamaa Neto et al., Phys. Plasmas 7, 2894 (2000). In this regime, the plasma detachment from the limiter is observed experimentally indicating that plasma recombination is the main process of plasma particle losses. In order to investigate if this process is actually taking place, measurements of electron temperature in the plasma are necessary. The electron temperature deduced from the plasma resistivity is in the range $0.2-1.5 \mathrm{eV}$.
\end{abstract}

\section{Introduction}

The runaway discharge is observed in TCABR tokamak with low-temperature weakly ionized plasma detached from the limiter [1]. This experimental fact can be caused only by volume recombination. Other distinctive feature of this regime is the relaxation instability with strong sawtooth relaxation of the line density. This phenomenon is explained by a short pulse, $\sim 10$ $\mu \mathrm{s}$, of plasma heating due to the instability, resulting in gas ionization. Then the instability is quenched, the plasma temperature drops, and the density decreases due to recombination. A value for the rate constant of recombination around $10^{-16} \mathrm{~m}^{3} / \mathrm{s}$ is deduced from the rate of density decay in the sawtooth relaxation.

It is not simple to explain the mechanism of recombination in this case. The usual three-body recombination provides the indicated value of the rate constant at temperatures less than $0.1 \mathrm{eV}$. However, energy balance calculations, which take into account only the collisional mechanism of the heat transfer from runaways to thermal electrons, lead to electron temperatures in the range $0.1-2 \mathrm{eV}$ for neutral densities $(0.1-1) \times 10^{19}$ $\mathrm{m}^{-3}$; the temperature decreases with the neutral density increase [2]. The molecular activated recombination (MAR), recently proposed in [3.4], provides the required rate constant. However, the MAR rate depends on vibrational excitation of hydrogen molecules. In our case, the plasma is rather transparent and molecules can effectively loose vibrational excitation due to collisions with the wall.

Thus, experimental data on electron temperature are important to understand the mechanism of recombination in our case. Unfortunately, usual diagnostics of the electron temperature are not applicable in this case due to the effect of runaway beam. In our previous work on TBR-1 tokamak, a method of electron temperature determination from the decay of loop voltage spikes has been developed [5]. Positive voltage spikes always occur in runaway discharges in TCABR during the relaxation instability. The results of voltage spikes analysis presented in this work give electron temperatures in the range $0.2-1.5 \mathrm{eV}$.

\section{Method}

The method of plasma resistivity determination from positive voltage spikes (VS) in runaway discharges is described in [5]. Let us denote the total toroidal current before the VS as $I_{t 0}=I_{b 0}+I_{p 0}$, where $I_{b 0}$ and $I_{p 0}$ are the runaway and plasma currents, respectively. We suppose in our model that some instability results in a stepshaped decrease in the runaway current, $I_{b 1}=I_{b 0}-\Delta I$ at $t=t_{1}$. In reply, the same but opposite plasma current is generated inductively, $I_{p 1}=I_{p 0}+\Delta I$, i.e. $I_{t 1}=I_{t 0}$ at $t=t_{1}$. Then plasma current decays to the equilibrium state due to resistive damping and inductive transformation to the runaway current. The electric field in a cylindrical plasma column is connected with the toroidal current density $j_{t}$ by the diffusion equation

$$
\frac{1}{\rho} \frac{\partial}{\partial \rho} \rho \frac{\partial E}{\partial \rho}=\mu_{0} \frac{\partial j_{t}}{\partial t} .
$$

The current density in Eq. (1), $j_{t}=j_{p}+j_{b}$, is the sum of the conductivity current (plasma current) $j_{p}=\sigma E$ and runaway current $j_{b}$ densities. Note that we consider only perturbative parts of current and electric field without special notations and put $t_{1}=0$.

A simplified analytical solution of Eq. (1) has the form: 


$$
E(\rho, t)=\sum_{n=1}^{\infty} A_{n} J_{0}\left(\beta_{n} \frac{\rho}{a}\right) \exp \left(-\gamma_{n} t\right)
$$

where

$$
\begin{gathered}
\gamma_{n}=\frac{g+\beta_{n}^{2}}{\mu_{0} \sigma a^{2}}, \\
g=\frac{\mu_{0} e^{2} a^{2} n_{b}}{m_{e} \gamma_{e f f}^{3}},
\end{gathered}
$$

$J_{0}$ is the Bessel function of the order zero, $\sigma$ is the plasma conductivity, $n_{b}$ is the runaway density and $\gamma_{e f f}$ is the effective relativistic factor for runaway beam, $\gamma_{e f f}=n_{b} / \int \gamma^{-3} f(p, t) d p, f$ is the distribution function of runaways. The $A_{n}$ 's are determined from the initial condition for electric field, and $\beta_{n}$ 's from the boundary condition. The solution (2) is obtained by the standard method of separation of variables assuming that the runaway density and plasma conductivity are constant in time and uniform on plasma cross section.

One can see in Eqs. (2)-(4) that pure resistive model corresponds to $g=0$.

As it was discussed in [5], the initial electric field distribution has a strong effect on the first phase of the VS, when the diffusion of the current is the main process. In the case of skinned initial electric field, i.e. when runaways are lost from some outer region of plasma column with thickness $\Delta_{0}<<a$, the initial loop voltage on the plasma boundary is

$$
V_{L}(a, 0)=\frac{R \cdot \Delta I}{a \Delta_{0} \sigma} .
$$

The characteristic time constants of VS can be estimated from Eq. (3) at the condition $g<<\beta_{n}^{2}$, which is valid in our case. According to Eqs. (2) and (3), the duration of the diffusion phase is determined by damping of the harmonic $n=2$. The time constant of current decay is determined by damping of the harmonic $n=1$,

$$
\tau_{d e c} \approx \frac{\mu_{0} \sigma a^{2}}{\beta_{1}^{2}}
$$

At typical tokamak parameters $R / a=3-6$, the relation $\beta_{n} \simeq 3 n-2$ can be used [5], i.e. $\tau_{\text {dif }} \approx 1 / 16 \tau_{\text {dec }}$ and the current decay during the diffusion phase is small. It increases somewhat when $\Delta_{0}$ decreases, but this effect is rather weak (see Fig. 2 in [5]). A comparison of the measured and calculated voltages allows us to infer an information about the plasma resistivity and initial electric field distribution.

In accordance with Eqs. (2)-(4), the runaway current generation results in a faster decay of the VS and a smaller decrease of the toroidal current, as compared to the prediction of the model which takes into account the resistive damping only. This effect is small, according to Eq. (3), if $g<<\beta_{1}^{2} \approx 1$.

\section{Analysis of Experimental Data}

The loop voltage is measured in TCABR by the voltage loops placed outside the vacuum vessel. The averaging on 8 loops distributed around of the vacuum vessel is used to decrease the effect of plasma shift. In general, the loop voltage is a result of toroidal currents changes:

$$
V_{L}=-M_{O H} \frac{d I_{O H}}{d t}-M_{v} \frac{d I_{v}}{d t}-\frac{d\left(M_{t} I_{t}\right)}{d t}-M_{s} \frac{d I_{s}}{d t}
$$

where $I_{O H}, I_{v}, I_{t}$, and $I_{s}$ are currents in ohmic heating and vertical field winding, toroidal plasma current, and toroidal eddy currents in structure respectively; $M_{O H}, M_{v}, M_{t}$, and $M_{s}$ are corresponding mutual inductances. We neglect in Eq. (7) the first two terms due to the weak coupling of the ohmic heating winding with plasma and the slow time response of the vertical field control system. In general, the inductance $M_{t}$ is a function of time due to the time variation of the current density distribution. Substantial simplifications are possible in the case of the short time duration of VS. The main element of TCABR structures producing an effect on magnetic measurements is the stainless steel vacuum vessel, with thickness $5-7 \mathrm{~mm}$, which has a time constant of eddy currents damping around $1 \mathrm{~ms}$. In the considered problem, the vacuum vessel plays a useful role of a magnetic field filter. The toroidal gap in the vacuum vessel allows to measure the total toroidal current changes without any time delay. For the fast events, $\Delta t<<1 \mathrm{~ms}$, the effect of variation in the inductance $M_{t}$ in Eq. (7) is suppressed. In result, the Eq. (7) can be used in the simplified form:

$$
V_{L}=M_{t} \frac{d I_{t}}{d t}
$$

with constant $M_{t}$, which is determined by the condition $\Psi=$ const on the vacuum vessel.

Typical behavior of the toroidal current and loop voltage in runaway discharges is shown in Figs. 1 and 2 for two shots with different neutral density. In shot 2998, the neutral gas pressure increases monotonically due to continuous gas puffing, with a rate $6.5 \times 10^{20}$ molecules $/ \mathrm{m}^{3} \mathrm{~s}$. In shot 3471 , the gas injection is cut off after the start up phase and neutrals exist only due to recycling. 


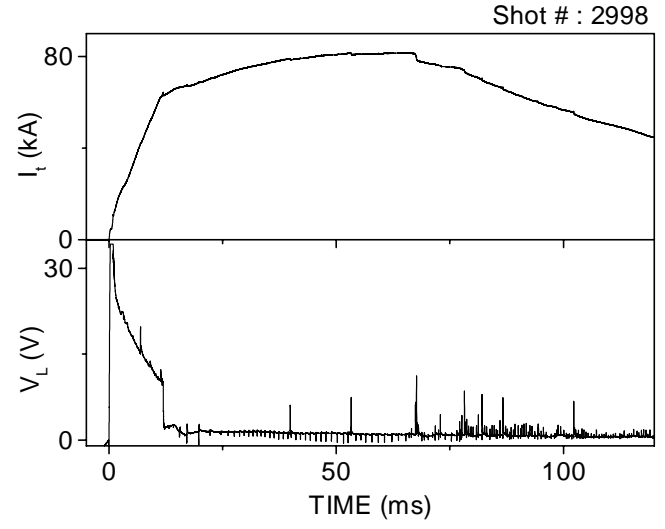

Figure 1a. Time evolution of the plasma current and loop voltage in runaway discharge 2998 . The gas puffing is used after $\mathrm{t}=23 \mathrm{~ms}$ with filling rate $6.5 \times 10^{20}$ molecules $/ \mathrm{m}^{3} \mathrm{~s}$.

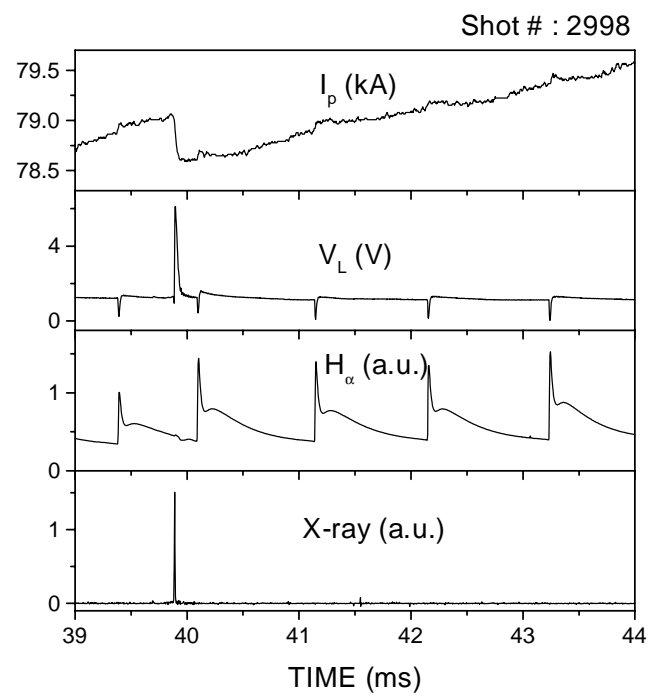

Figure 1b. Expanded spikes in the plasma current, loop voltage, $\mathrm{H}_{\alpha}$ and x-ray emissions in shot 2998 .

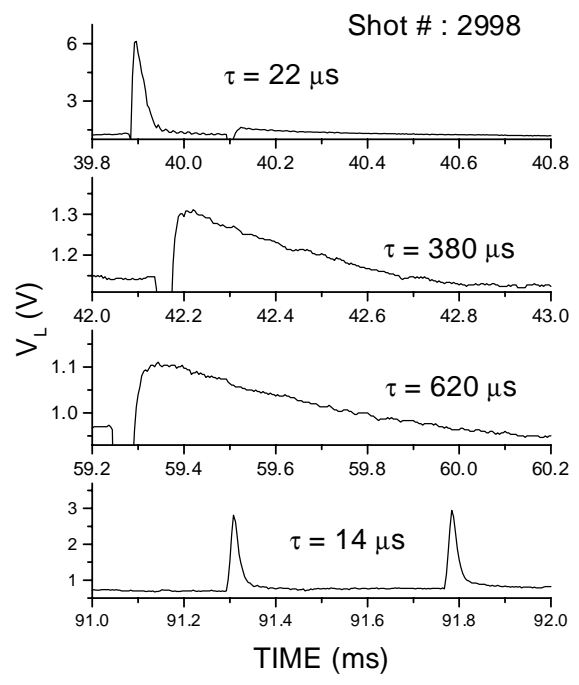

Figure 1c. Expanded voltage spikes in shot 2998 for different moments of time; the time constant of voltage decay is indicated.

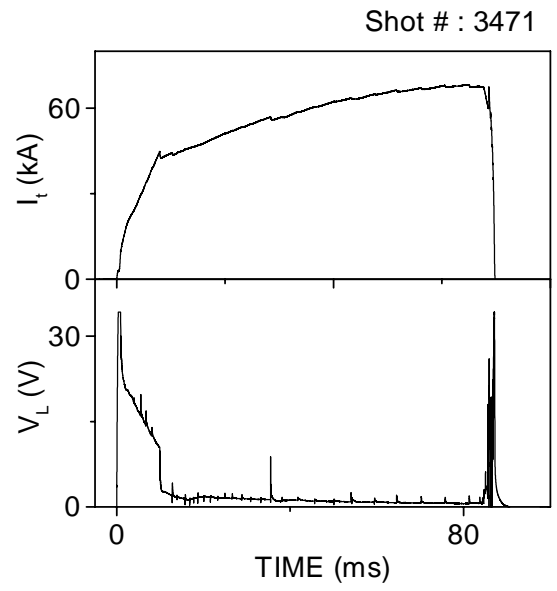

Figure 2a. Time evolution of the plasma current and loop voltage in runaway discharge 3471 . The gas puffing is cut off after the current ramp-up at $t=23 \mathrm{~ms}$.

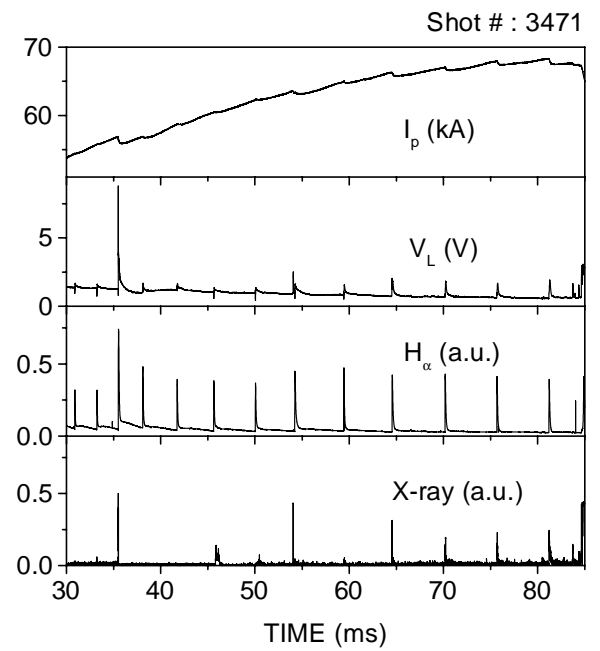

Figure 2b. Expanded spikes in the plasma current, loop voltage, $\mathrm{H}_{\alpha}$ and $\mathrm{x}$-ray emissions in shot 3471 .

Shot \# : 3471

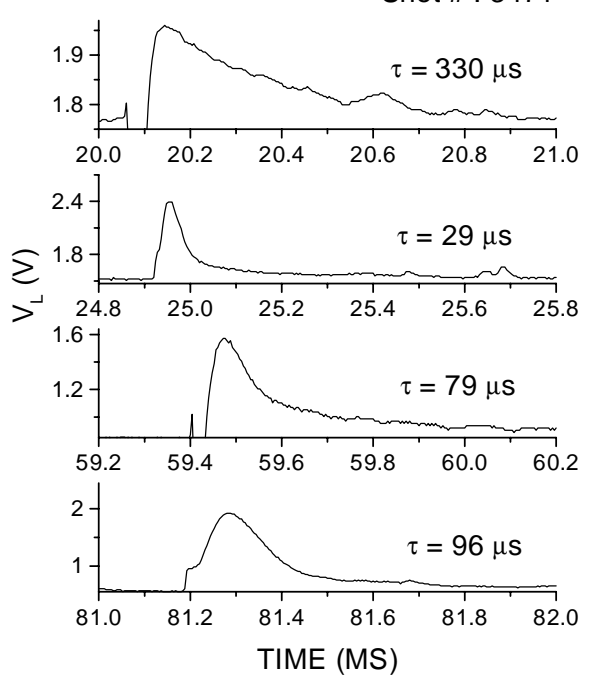

Figure 2c. Expanded voltage spikes in shot 3471 for different moments of time; the time constant of voltage decay is indicated. 
Two types of the VS are observed (see Fig. 1b ). In the first type, they occur always simultaneously with the $\mathrm{H}_{\alpha}$ spikes and are clearly associated with relaxation instability described in [1]. The VS is usually first negative followed by a positive overshoot with amplitude of around $0.1 \mathrm{~V}$. In some cases, the VS of this type are mostly positive and have larger amplitude, as one can see in Fig. $2 \mathrm{~b}$. In the second type, as seen at $t \approx 40$ $\mathrm{ms}$ in Fig. 1b, the spikes are not associated with the relaxation instability. They are only (or mostly) positive and have rather high amplitude, up to $10 \mathrm{~V}$ or more, but $\mathrm{H}_{\alpha}$ spikes related to the VS of this type are relatively small or even absent as in the case shown in Fig. 1b.

According to Eqs. ( 3 ) and (4), the effect of runaway current generation on the voltage spike decay is small if $g<<1$. To estimate the value of $g$, the runaway density is calculated as $n_{b}=I_{b} / e c \pi a_{b}^{2}$; we use $I_{b}=I_{t}$ because the plasma current is negligible small at our parameters. The average energy of runaways is deduced from the measured values of the Shafranov parameter as 2-4 MeV, i.e. $\gamma_{e f f}=5-9$. This gives $g=0.15-0.03$, i.e., we can neglect this effect.

Examples of the expanded VS are presented in Figs. $1 c$ and $2 c$ with indication of the time constants of the VS decay. One can see strong differences between small and large-amplitude spikes regarding these time constants. Small spikes have rather large time constant, typically 300-600 $\mu \mathrm{s}$. However, corresponding spikes in the toroidal current, which have to be in this case around $100 \mathrm{~A}$, in accordance to Eq. (8), are poorly measurable, as one can see in Fig. 1b. A more careful analysis indicates inward plasma shifts of around 0.1 $\mathrm{cm}$ correlated with the VS. According to estimations, such shift may be responsible for the positive VS with amplitude of around $0.1 \mathrm{~V}$. Therefore, larger VS are preferable for this diagnostic. In this case, spikes in the current are also measurable, as one can see in Fig. $2 \mathrm{~b}$ and also in Fig. 3 where other examples of large positive VS are presented. Note that the time constant of the current decay is substantially lower that of the voltage spike, as one can see in Fig. 3. In the case of voltage spikes with time constant much less than that of the vacuum vessel $(\sim 1 \mathrm{~ms})$ this fact can not be caused by the effect of plasma shift. The limitation of the time response of the loop voltage, $\approx 10 \mu \mathrm{s}$, and the time resolution of the data acquisition system, $4 \mu \mathrm{s}$, can not also explain substantial difference between the voltage and current spikes caused with some systematic error in measurements, which are not clear for us at present.

The plasma conductivity is determined from the time constant according to Eq. (6). Using the Spitzer formula for plasma conductivity, the electron temperature is calculated as

$$
T_{e}[\mathrm{eV}]=\left(5.23 \times 10^{-5} Z_{i} \sigma \ln \Lambda\right)^{2 / 3}
$$

where $Z_{i}$ is the effective ion charge and $\ln \Lambda$ is the Coulomb logarithm. A dependence of the electron tem- perature on the time constant is shown in Fig. 4. Here we use the plasma minor radius as the parameter. From the line density measurements by the interferometer along different vertical chords, the minor plasma radius is estimated to be in the range $0.09 \mathrm{~m} \leq a \leq 0.14$ $\mathrm{m}$. For plasma radius $0.14 \mathrm{~m}$ and $Z_{i}=1$, this method gives $T_{e}=0.2-1.5 \mathrm{eV}$ for the measured values, $10-100$ $\mu s$, of the time constant of VS decay. Qualitatively, the temperature increases with the decrease of the neutral density in agreement with results of the energy balance calculations [2].

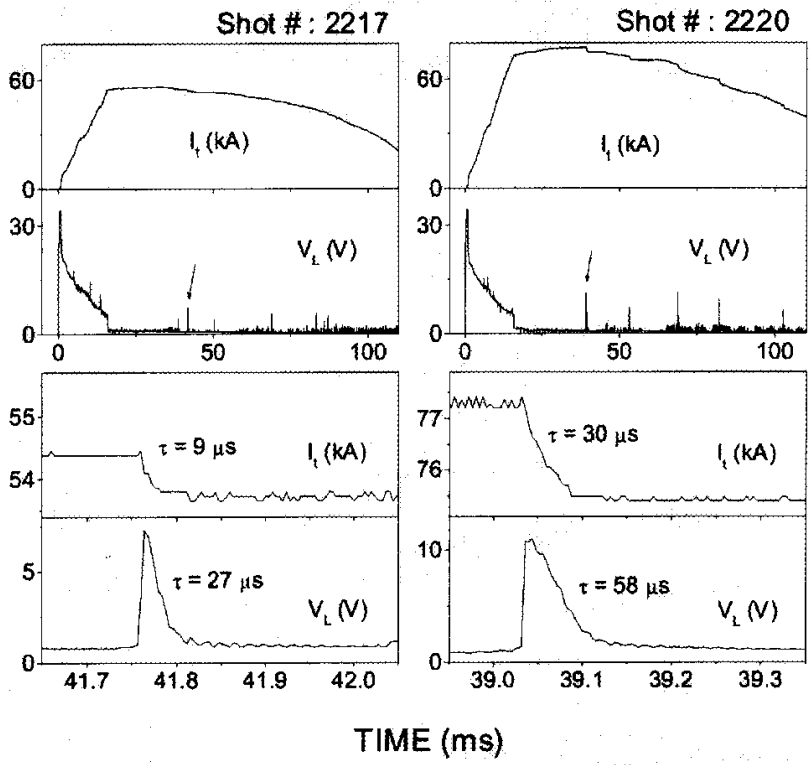

Figure 3. Dependence of the plasma electron temperature on the time constant of the voltage decay obtained from Eqs. (6) and (9)

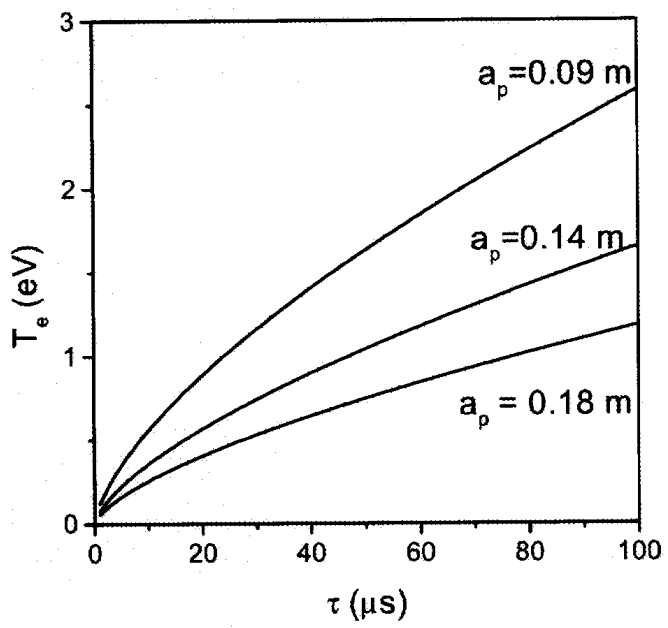

Figure 4. Examples of large positive voltage spikes. The current changes are measurable in this case. The time constants of the voltage and current decay are indicated. 


\section{Conclusion}

The plasma resistance is determined from voltage spike decay in runaway discharge in TCABR tokamak. The electron temperature, calculated from the resistance using Spitzer formula, is found to be in the range $0.2-1.5$ $\mathrm{eV}$. These values of $\mathrm{T}_{e}$ are in reasonable agreement with those obtained from energy and particle analysis [2].

If these measurements are correct, one could conclude that molecular activated recombination (MAR) is the main process of plasma losses in this case. However, it is difficult to accept this conclusion. According to the theory [3.4] the MAR requires vibrational excitation of hydrogen molecules. In our case, the plasma is rather transparent and molecules can effectively loose vibrational excitation due to collisions with the wall. Therefore, other methods of plasma temperature measurements are highly desirable.

\section{Acknowledgment}

This work has been supported by FAPESP and by the Brazilian Ministry of Science and Technology through the PRONEX Projects.

\section{References}

[1] R.M.O. Galvão, Yu.K. Kuznetsov, I.C. Nascimento et al., Plasma Phys. Control. Fusion 43, 1181 (2001).

[2] T.K. Soboleva, R.M.O. Galvão, S.I. Krasheninnikov, Yu.K. Kuznetsov and I.N. Nascimento, submitted for publication in Braz. J. Phys.

[3] S.I. Krasheninnikov, A. Yu. Pigarov, and D.J. Sigmar, Phys. Letters A214, 295 (1996).

[4] A.Yu. Pigarov and S.I. Krasheninnikov, Phys. Letters A222, 251 (1996).

[5] I. El Chamaa Neto, Yu.K. Kuznetsov, I.C. Nascimento, R.M.O. Galvão, and V.S. Tsypin, Phys. Plasmas 7, 2894 (2000). 